\title{
Stability of spherically symmetric timelike thin-shells in general relativity with a variable equation of state
}

\author{
S. Habib Mazharimousavi * M. Halilsoy ${ }^{\dagger}$ and S. N. Hamad Amen \\ Department of Physics, Eastern Mediterranean University, Gazimag̃usa, Turkey.
}

(Dated: July 19, 2018)

\begin{abstract}
We study spherically symmetric timelike thin-shells in $3+1$-dimensional bulk spacetime with a variable equation of state for the fluid presented on the shell. In such a fluid the angular pressure $p$ is a function of both surface energy density $\sigma$ and the radius $R$ of the thin-shell. Explicit cases of the thin shells connecting two non-identical cloud of strings spacetimes and a flat Minkowski spacetime to the Schwarzschild metric are investigated.

PACS numbers:

Keywords: Thin-shell; Spherically symmetric; Stability; Variable Equation of state
\end{abstract}

\section{INTRODUCTION}

Time-like thin-shells in spherically symmetric static spacetimes are the most interesting cosmological object which can be constructed in general relativity. Such models of cosmological objects have been used to analyze some astrophysical phenomena such as gravitational collapse and supernova explosions. The seminal work of Israel in 1966 [1, 2] provided a concrete formalism for constructing the time-like shells, in general, by gluing two different manifolds at the location of the thin-shell. As it was shown in [1, although the metric tensor of the shell which is induced by the bulk spacetime presented in both sides of the shell must be continuous, the extrinsic curvature across the shell is not continuous and therefore matter has to be introduced on the shell. This formalism has been employed to study shells in general relativity by many authors for which a good review paper has been worked out by Kijowski et al in Ref. 3]. In 1990 an exact solution for a static shell which surrounds a black hole was found by Frauendiener et al [4] and its stability was also studied in Refs. [5] and 6]. In the formalism introduced by Israel there are some conditions which are called Israel junction conditions. These conditions provide a systematic method of finding the energy momentum tensor presented on the shell. In 7 a computer program was prepared to apply the junction conditions on the thin-shells in general relativity using computer algebra. Models of stars and circumstellar shells in general relativity were studied in $[8$. In $[9,10$ the stability of spherically symmetric thin-shells was studied while the gravitational collapse of thin-shells was considered in [11] and [12. Thin-shells in Gauss-Bonnet theory of gravity has been studied in 13 while the rotating thin-shells has been introduced in Ref. 14. Stability of charged thin-shells was considered in [15] and its collapse in isotropic coordinates was investigated in [16. In [17] charge screening by thin-shells in a $2+1$-dimensional regular black hole has been studied while the thermodynamics, entropy, and stability of thin-shells in 2+1-dimensional flat spacetimes have been given in 18 and 19 . Recently in 20 the stability of thin-shell interfaces inside compact stars has been studied by Pereira et al which is very interesting as they consider a compact star with the core and the crust with different energy momentum tensors and consequently with different metric tensors. Screening of the Reissner-Nordström charge by a thin-shell of dust matter has also been introduced recently in [21. Finally one of the recent works published in this context is about thin-shells joining local cloud of strings geometries [22].

In this study first we give a brief review of the thin-shell formalism and following that we employ a variable equation of state (EoS) for the perfect fluid presented on the surface of the thin-shell of the form $p=\psi(\sigma, R)$ where $p$ is the surface pressure, $\sigma$ is the surface energy density and $R$ is the radius of the shell. Let's add that the function $\psi$ must be differentiable with respect to its arguments $R$ and $\sigma$. This model of EoS has been introduced first in [23] and was used recently in 24]. Following the generic stability analysis with a variable EoS we study two specific cases including a thin-shell connecting two spacetimes of different cloud of strings and a vacuum flat Minkowski spacetime connected to a Schwarzschild metric.

\footnotetext{
*Electronic address: habib.mazhari@emu.edu.tr

${ }^{\dagger}$ Electronic address: mustafa.halilsoy@emu.edu.tr

‡Electronic address: sarbaz256@gmail.com
} 


\section{SPHERICALLY SYMMETRIC TIMELIKE THIN-SHELLS}

In $3+1$-dimensional spherically symmetric bulk spacetime a $2+1$-dimensional timelike thin-shell divides the spacetime into two parts which we shall call as the inside and outside of the shell. The line elements of the spacetimes in different sides must naturally be different otherwise the thin-shell becomes a trivial invisible object. Let's label the spacetime inside the shell as 1 and outside the shell as 2 . Hence the line element of each side may be written generically as

$$
d s_{a}^{2}=-f_{a}\left(r_{a}\right) d t_{a}^{2}+\frac{d r_{a}^{2}}{f_{a}\left(r_{a}\right)}+r_{a}^{2}\left(d \theta_{a}^{2}+\sin ^{2} \theta_{a} d \varphi_{a}^{2}\right)
$$

in which $a=1,2$ for inside and outside, respectively. We add that in general the coordinates i.e., $t_{a}, r_{a}, \theta_{a}$ and $\varphi_{a}$ need not be the same. In general a thin-shell is a constraint condition on the coordinates of the bulk spacetime but in our study the thin-shell is defined by $F:=r_{a}-R(\tau)=0$ in which $\tau$ is the proper time measured by an observer on the shell such that on both sides we define

$$
-f_{a}(R)\left(\frac{d t_{a}}{d \tau}\right)^{2}+\frac{1}{f_{a}(R)}\left(\frac{d R}{d \tau}\right)^{2}=-1
$$

As one of the Israel junction condition, $d s_{(t s)}^{2}$ from one side to the other of the thin-shell must be continuous. Hence, the coordinates on the shell i.e., $\tau, \theta_{a}$ and $\varphi_{a}$ have to be identical on both sides so that we shall remove the sub index $a$. This results in a unique induced metric on the shell which is applicable to both sides expressed by

$$
d s_{(t s)}^{2}=-d \tau^{2}+R^{2}(\tau)\left(d \theta^{2}+\sin ^{2} \theta d \varphi^{2}\right) .
$$

Before we proceed further let us note that although the proper time in different sides of the shell is common the coordinate times $t_{a}$ are different and they are found from (3) as

$$
\dot{t}_{a}^{2}=\frac{f_{a}(R)+\dot{R}^{2}}{f_{a}^{2}(R)}
$$

in which a dot stands for the derivative with respect to the proper time $\tau$. Let's also add that $t_{1}$ and $t_{2}$ refer to the coordinate times of the inner and outer regions.

For future use we set our coordinate systems of the bulk i.e., $d s_{a}^{2}=g_{\mu \nu}^{(a)} d x^{(a) \mu} d x^{(a) \nu}$ and the shell i.e., $d s_{(t s)}^{2}=$ $h_{i j} d \xi^{i} d \xi^{j}$ as follow: for the bulk spacetime $x^{(a) \mu}=\left\{t_{a}, r_{a}, \theta_{a}, \varphi_{a}\right\}$ and $\xi^{i}=\{\tau, \theta, \varphi\}$ for the thin-shell. The second fundamental form (or extrinsic curvature) tensor of the shell in each side can be found as

$$
K_{i j}^{(a)}=-n_{\gamma}^{(a)}\left(\frac{\partial^{2} x^{(a) \gamma}}{\partial \xi^{i} \partial \xi^{j}}+\Gamma_{\alpha \beta}^{(a) \gamma} \frac{\partial x^{(a) \alpha}}{\partial \xi^{i}} \frac{\partial x^{(a) \beta}}{\partial \xi^{j}}\right)
$$

in which $n_{\gamma}^{(a)}$ is the four-normal spacelike vector on each side of the thin-shell pointing outward given by

$$
n_{\gamma}^{(a)}=\left(-\dot{R}(\tau), \dot{t}_{a}, 0,0\right)
$$

The nonzero components of the extrinsic curvature, therefore, are found to be

$$
\begin{gathered}
K_{\tau \tau}^{(a)}=-\frac{2 \ddot{R}(\tau)+f_{a}^{\prime}(R)}{2 \sqrt{f_{a}(R)+\dot{R}^{2}}}, \\
K_{\theta \theta}^{(a)}=R(\tau) \sqrt{f_{a}(R)+\dot{R}^{2}}
\end{gathered}
$$

and

$$
K_{\varphi \varphi}^{(a)}=R(\tau) \sqrt{f_{a}(R)+\dot{R}^{2}} \sin ^{2} \theta
$$

One observes that unlike the first fundamental form, the second fundamental form is not continuous in general. However, if $f_{a}(R)$ and $f_{a}^{\prime}(R)$ are the same on both sides of the shell then $K_{i j}$ as well as $h_{i j}$ are both continuous. In case that $K_{i j}$ is not continuous it satisfies the other Israel junction condition

$$
\left[K_{i}^{j}\right]-\delta_{i}^{j}[K]=-8 \pi G S_{i}^{j}
$$


in which $\left[K_{i}^{j}\right]=K_{i}^{(2) j}-K_{i}^{(1) j},[K]=\operatorname{trac}\left[K_{i}^{j}\right]=\left[K_{i}^{i}\right]$ and

$$
S_{i}^{j}=\operatorname{diag}(-\sigma, p, p)
$$

is the energy-momentum tensor of the thin-shell. Herein, $\sigma$ is the energy density and $p$ the angular pressure on the shell. We note that, as we have considered the bulk to be spherically symmetric, the pressures in $\theta$ and $\varphi$ directions are identical and the energy-momentum tensor is of a perfect fluid type. The explicit form of the $\sigma$ and $p$ are given by

$$
\sigma=-\frac{1}{4 \pi G}\left(\frac{\sqrt{f_{2}(R)+\dot{R}^{2}}-\sqrt{f_{1}(R)+\dot{R}^{2}}}{R(\tau)}\right)
$$

and

$$
p=\frac{1}{8 \pi G}\left(\frac{2 \ddot{R}(\tau)+f_{2}^{\prime}(R)}{2 \sqrt{f_{2}(R)+\dot{R}^{2}}}-\frac{2 \ddot{R}(\tau)+f_{1}^{\prime}(R)}{2 \sqrt{f_{1}(R)+\dot{R}^{2}}}+\frac{\sqrt{f_{2}(R)+\dot{R}^{2}}-\sqrt{f_{1}(R)+\dot{R}^{2}}}{R(\tau)}\right) .
$$

In the case of a static thin-shell one has to set $R(\tau)=R_{0}$ which is a constant and consequently

$$
\sigma_{0}=-\frac{1}{4 \pi G}\left(\frac{\sqrt{f_{2}\left(R_{0}\right)}-\sqrt{f_{1}\left(R_{0}\right)}}{R_{0}}\right)
$$

and

$$
p_{0}=\frac{1}{8 \pi G}\left(\frac{f_{2}^{\prime}\left(R_{0}\right)}{2 \sqrt{f_{2}\left(R_{0}\right)}}-\frac{f_{1}^{\prime}\left(R_{0}\right)}{2 \sqrt{f_{1}\left(R_{0}\right)}}+\frac{\sqrt{f_{2}\left(R_{0}\right)}-\sqrt{f_{1}\left(R_{0}\right)}}{R_{0}}\right) .
$$

\section{STABILITY}

Let's assume that our constructed thin-shell is at equilibrium at $R=R_{0}$ which means $\dot{R}=\ddot{R}=0$ and therefore the energy density and the pressures are given by Eqs. (14) and (15). Any radial perturbation causes the radius of the shell to be changed in a dynamical sense. In other words after the radial perturbation $R$ becomes a function of proper time $\tau$ and consequently the energy density and the angular pressures are found to be Eqs. (12) and (13) which also satisfy

$$
\frac{d \sigma}{d R}+\frac{2}{R}(p+\sigma)=0 .
$$

This equation is the dynamic relation that connects $p$ and $\sigma$ after the perturbation. Furthermore, any kind of fluid presented on the shell has to satisfy an equation of state which is nothing but a relation between $p$ and $\sigma$. This relation is traditionally expressed as

$$
p=p(\sigma)
$$

but in our study we use a more general EoS given by [23, 24]

$$
p=\psi(R, \sigma) .
$$

A substitution in (16) yields

$$
\frac{d \sigma}{d R}+\frac{2}{R}(\psi(R, \sigma)+\sigma)=0
$$

which is a principal equation that connects $\sigma$ to $R$ after the perturbation. In addition to this, from the explicit form of $\sigma$ in Eq. (12) we find

$$
\dot{R}^{2}+V(R, \sigma(R))=0
$$


in which

$$
V(R, \sigma(R))=\frac{f_{1}(R)+f_{2}(R)}{2}-\frac{\left(f_{1}(R)-f_{2}(R)\right)^{2}}{(8 \pi G R \sigma(R))^{2}}-(2 \pi G R \sigma(R))^{2} .
$$

This equation is a one dimensional equation of motion for the radius of the thin-shell after the perturbation. Together with Eq. (19) gives a clear picture of the motion of the thin-shell after the perturbation. More precisely, the solution of Eq. (19) is used in (20) and the general motion of the radius of the thin-shell, in principle is found by solving Eq. (20). The nature of the motion after the perturbation depends on the form of the function $\psi(R, \sigma)$ given by EoS and the metric functions $f_{1}(R)$ and $f_{2}(R)$. We comment that the general one-dimensional equation of motion (20) is highly non-linear. In general we do not expect an exact, closed form solution for the radius of the thin-shell after the perturbation. A linearized version of this equation helps us to know the general behaviour of the motion of the thin-shell after the perturbation without going through the complete solution. As we have stated the thin-shell is in equilibrium at $R=R_{0}$ so we expand $V(R, \sigma(R))$ about $R=R_{0}$ and keep it at the first nonzero term. This is called a linearized radial perturbation. The expansion of $V(R, \sigma(R))$ about $R=R_{0}$ reads as

$$
V(R, \sigma)=V\left(R_{0}, \sigma_{0}\right)+\left.\frac{d V}{d R}\right|_{R=R_{0}}\left(R-R_{0}\right)+\left.\frac{1}{2} \frac{d^{2} V}{d R^{2}}\right|_{R=R_{0}}\left(R-R_{0}\right)^{2}+\mathcal{O}\left(\left(R-R_{0}\right)^{3}\right)
$$

where $V\left(R_{0}, \sigma_{0}\right)$ and $\left.\frac{d V}{d R}\right|_{R=R_{0}}$ both are zero identically i.e., the first because $\dot{R}_{0}^{2}=0$ and the second because $R=R_{0}$ is the equilibrium radius in the sense that the force is zero there. Introducing $x=R-R_{0}$, up to the second order we get

$$
\dot{x}^{2}+\omega^{2} x^{2} \simeq 0
$$

in which

$$
\omega^{2}=\left.\frac{1}{2} \frac{d^{2} V}{d R^{2}}\right|_{R=R_{0}}
$$

Derivative with respect to $\tau$ implies

$$
\ddot{x}+\omega^{2} x \simeq 0
$$

which clearly for $\omega^{2}>0$ represents an oscillation about $x=0$. This, however, means the radius of the thin-shell oscillates about the equilibrium radius $R=R_{0}$. This is what we mean by a stable condition. In other words if

$$
\left.\frac{1}{2} \frac{d^{2} V}{d R^{2}}\right|_{R=R_{0}}>0
$$

the thin-shell oscillates and remains stable. Unlike $\omega^{2}>0$ if $\omega^{2}<0$ then the motion of the radius of the thin-shell becomes an exponential form which yields an unstable thin-shell. To proceed further we need to calculate $\left.\frac{d^{2} V}{d R^{2}}\right|_{R=R_{0}}$ and as $V=V(R, \sigma)$ we shall need $\sigma^{\prime}=\frac{d \sigma}{d R}$ and $\sigma^{\prime \prime}=\frac{d^{2} \sigma}{d R^{2}}$. Upon differentiation with respect to $R$, (19) yields

$$
\sigma^{\prime \prime}=\frac{2}{R^{2}}(\psi+\sigma)\left(2 \frac{\partial \psi}{\partial \sigma}+3\right)-\frac{2}{R} \frac{\partial \psi}{\partial R} .
$$

Next, we find $V^{\prime \prime}(R)$ at $R=R_{0}$ by applying $\sigma^{\prime}$ and $\sigma^{\prime \prime}$ whenever we need them which becomes

$$
\begin{gathered}
V_{0}^{\prime \prime}=-\frac{16 \pi G F_{0} H_{0}}{F_{0}-H_{0}} \psi_{, R}+\frac{2\left[H_{0}\left(2 F_{0}^{2}-f_{10}^{\prime} R_{0}\right)-F_{0}\left(2 H_{0}^{2}-f_{20}^{\prime} R_{0}\right)\right]}{(F-H) R_{0}^{2}} \psi_{, \sigma}+ \\
\frac{\left[4 F_{0}^{4}-2 R_{0}\left(f_{10}^{\prime}+R_{0} f_{10}^{\prime \prime}\right) F_{0}^{2}+R_{0}^{2} f_{10}^{\prime 2}\right] H_{0}^{3}-\left[4 H_{0}^{4}-2 R_{0}\left(f_{20}^{\prime}+R_{0} f_{20}^{\prime \prime}\right) H_{0}^{2}+R_{0}^{2} f_{20}^{\prime 2}\right] F_{0}^{3}}{2\left(F_{0}-H_{0}\right) F_{0}^{2} H_{0}^{2} R_{0}^{2}}
\end{gathered}
$$

in which $F_{0}=\sqrt{f_{10}}, H_{0}=\sqrt{f_{20}}, \psi_{, R}=\left.\frac{\partial \psi}{\partial R}\right|_{R=R_{0}}$ and $\psi_{, \sigma}=\left.\frac{\partial \psi}{\partial \sigma}\right|_{R=R_{0}}$. 


\section{THIN-SHELL CONNECTING TWO SPACETIMES OF CLOUD OF STRINGS}

In this section we consider $f_{1}=\kappa_{1}$ and $f_{2}=\kappa_{2}$ in which $\kappa_{1}$ and $\kappa_{2}$ are two positive constants satisfying $\kappa_{1}>\kappa_{2}$. Such spacetimes where $\kappa_{1}, \kappa_{2} \neq 1$ represent the so called cloud of strings spacetime [22, 25] 28]. The energy momentum tensor at the equilibrium is given by

$$
S_{i}^{j}=-\frac{\sqrt{\kappa_{1}}-\sqrt{\kappa_{2}}}{4 \pi G R_{0}} \operatorname{diag}\left(1,-\frac{1}{2},-\frac{1}{2}\right)
$$

which means

$$
\sigma_{0}=\frac{\sqrt{\kappa_{1}}-\sqrt{\kappa_{2}}}{4 \pi G R_{0}}
$$

and

$$
p_{0}=-\frac{1}{2} \sigma_{0}
$$

This energy momentum tensor satisfies the weak energy condition which states that $\sigma_{0} \geq 0$, and $\sigma_{0}+p_{0} \geq 0$ and therefore it is physical. To proceed with the stability analysis we must choose an EoS. For the first choice we set

$$
\frac{\partial \psi}{\partial R}=\omega_{1}
$$

and

$$
\frac{\partial \psi}{\partial \sigma}=\omega_{2}
$$

in which both $\omega_{1}$ and $\omega_{2}$ are constants. The general form of $V^{\prime \prime}\left(R_{0}\right)$ given in Eq. (28) yields

$$
V^{\prime \prime}\left(R_{0}\right)=-\frac{2 \sqrt{\kappa_{1} \kappa_{2}}\left[8 G \pi \omega_{1} R_{0}^{2}-\left(2 \omega_{2}+1\right)\left(\sqrt{\kappa_{1}}-\sqrt{\kappa_{2}}\right)\right]}{R_{0}^{2}\left(\sqrt{\kappa_{1}}-\sqrt{\kappa_{2}}\right)} .
$$

In order to have $V^{\prime \prime}\left(R_{0}\right)>0$ one must impose

$$
8 G \pi \omega_{1} R_{0}^{2}-\left(2 \omega_{2}+1\right)\left(\sqrt{\kappa_{1}}-\sqrt{\kappa_{2}}\right)<0
$$

which in turn implies that

$$
\omega_{1}<\frac{\left(\sqrt{\kappa_{1}}-\sqrt{\kappa_{2}}\right)}{8 G \pi R_{0}^{2}}\left(2 \omega_{2}+1\right)
$$

The case where $\omega_{1}=0$ implies a linear perfect fluid which is stable for $\omega_{2}>-\frac{1}{2}$ and is unstable for $\omega_{2}<-\frac{1}{2}$. For the case where $\omega_{1} \neq 0$ the situation becomes more complicated. In Fig. 1 we plot

$$
\omega_{1}=\frac{\left(\sqrt{\kappa_{1}}-\sqrt{\kappa_{2}}\right)}{8 G \pi R_{0}^{2}}\left(2 \omega_{2}+1\right)
$$

for $\frac{\left(\sqrt{\kappa_{1}}-\sqrt{\kappa_{2}}\right)}{8 G \pi R_{0}^{2}}=0.1,0.2,0.3$ and 0.4. As it is imposed from the condition (35) the values of $\omega_{1}$ and $\omega_{2}$ under the lines for each specific choice of $\frac{\left(\sqrt{\kappa_{1}}-\sqrt{\kappa_{2}}\right)}{8 G \pi R_{0}^{2}}$ yields a region of stability while the opposite side (above the lines) stands for the values of $\omega_{1}$ and $\omega_{2}$ that result in an unstable thin-shell. In Ref. 22] thin-shells joining local cloud of strings geometries has been studied by Eiroa et al. Although their line elements for the cloud of strings geometries was written in static axially symmetric form and their equation of state was linear perfect fluid, their results is in agreement with what we found here in Eq. (36) provided $\omega_{1}=0$. Let's add that $\omega_{1}<0$ increases the region of stability in comparison with the linear perfect fluid with $\omega_{1}=0$. This can be seen from Eq. (36) and in Fig. 1. 


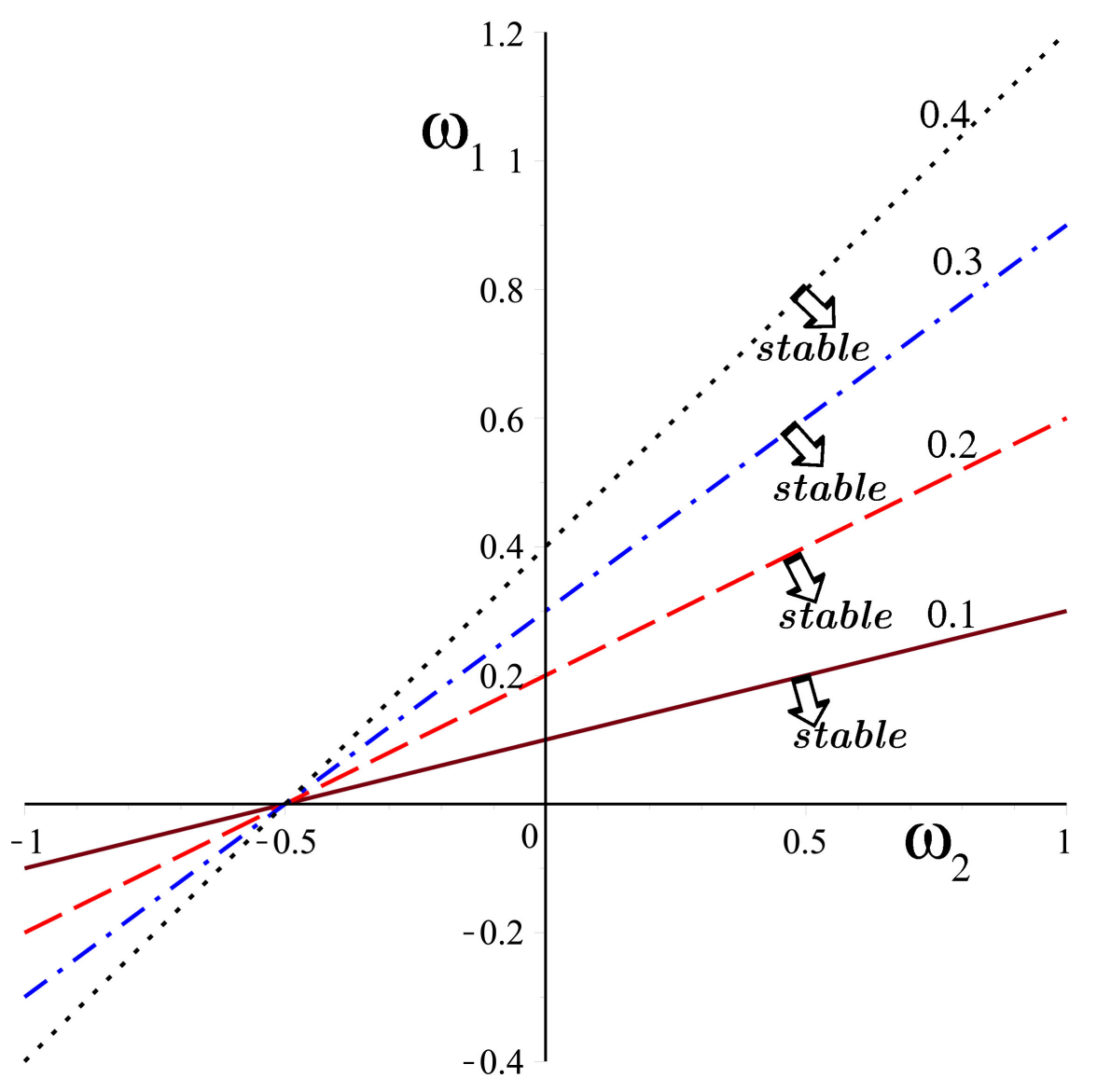

FIG. 1: A plot of $\omega_{1}$ with respect to $\omega_{2}$ for various values of $\frac{\left(\sqrt{\kappa_{1}}-\sqrt{\kappa_{2}}\right)}{8 G \pi R_{0}^{2}}=0.1,0.2,0.3$ and 0.4 . The arrows show the region of stability while the opposite side is the unstable zone for each case.

\section{THIN-SHELL CONNECTING VACUUM TO SCHWARZSCHILD}

In this part we consider the inner spacetime to be flat with $f_{1}=1$ and the outer spacetime the Schwarzschild with $f_{2}\left(r_{2}\right)=1-\frac{2 m}{r_{2}}$. The closed forms of $\sigma_{0}$ and $p_{0}$ are found to be

$$
\sigma_{0}=\frac{1-\sqrt{1-\frac{2 m}{R_{0}}}}{4 \pi G R_{0}}
$$

and

$$
p_{0}=-\frac{m-R_{0}+R_{0} \sqrt{1-\frac{2 m}{R_{0}}}}{8 \pi G R_{0}^{2} \sqrt{1-\frac{2 m}{R_{0}}}} .
$$

These clearly satisfy the weak energy conditions i.e., $\sigma_{0} \geq 0$ and $\sigma_{0}+p_{0} \geq 0$ provided $R_{0}>2 m$. Furthermore, one finds

$$
V_{0}^{\prime \prime}=\frac{16 G \pi \Omega}{\Omega-1} \psi_{, R}+\frac{2\left(2 \psi_{, \sigma}+1\right) \Omega}{R_{0}^{2}}
$$

in which $\Omega=\sqrt{1-\frac{2 m}{R_{0}}}>0, \psi_{, R}=\left.\frac{\partial \psi}{\partial R}\right|_{R=R_{0}}$ and $\psi_{, \sigma}=\left.\frac{\partial \psi}{\partial \sigma}\right|_{R=R_{0}}$. In this case also we set $\frac{\partial \psi}{\partial R}=\omega_{1}$ and $\frac{\partial \psi}{\partial \sigma}=\omega_{2}$ which implies

$$
V_{0}^{\prime \prime}=-\frac{16 G \pi \Omega}{1-\Omega} \omega_{1}+\frac{2\left(2 \omega_{2}+1\right) \Omega}{R_{0}^{2}} .
$$


For the case $\omega_{1}=0$ which corresponds to a linear perfect fluid one finds $V_{0}^{\prime \prime} \geq 0$ with $2 \omega_{2}+1 \geq 0$ or equivalently $\omega_{2} \geq-\frac{1}{2}$. For the case $\omega_{1} \neq 0$ we have to work out the regions in the plane of $\omega_{1}$ and $\omega_{2}$ such that $V_{0}^{\prime \prime} \geq 0$. To find the region where $V_{0}^{\prime \prime} \geq 0$ we find $\omega_{2}$ in terms of $\omega_{1}$ such that $V_{0}^{\prime \prime}=0$. This amounts to

$$
\omega_{2}=-\frac{1}{2}+\chi \omega_{1}
$$

in which

$$
\chi=\frac{4 \pi G R_{0}^{2}}{1-\sqrt{1-\frac{2 m}{R_{0}}}} .
$$

Depending on the value of $m$ and $R_{0}>2 m$, one finds

$$
4 \pi G R_{0}^{2}<\chi<\infty .
$$

In Fig. 2 we plot $\omega_{2}$ versus $\omega_{1}$ for various values for $\chi=0.1,0.2,0.3$ and 0.4 . Also the stability regions for each case is shown by an arrow indicator. We would like to add that such a thin-shell has been indirectly considered by Eiroa and Simeone in [15] with a linear perfect fluid. In both Fig. 1 and 2 in [15], the upper left, represents the thin-shell connecting the vacuum to Schwarzschild with their parameter $\eta$. Presence of $\omega_{1}$ changes the stability regime, especially if $\omega_{1}<0,(41)$ implies that the term due to $\omega_{1}$ is positive which increases the stability region.

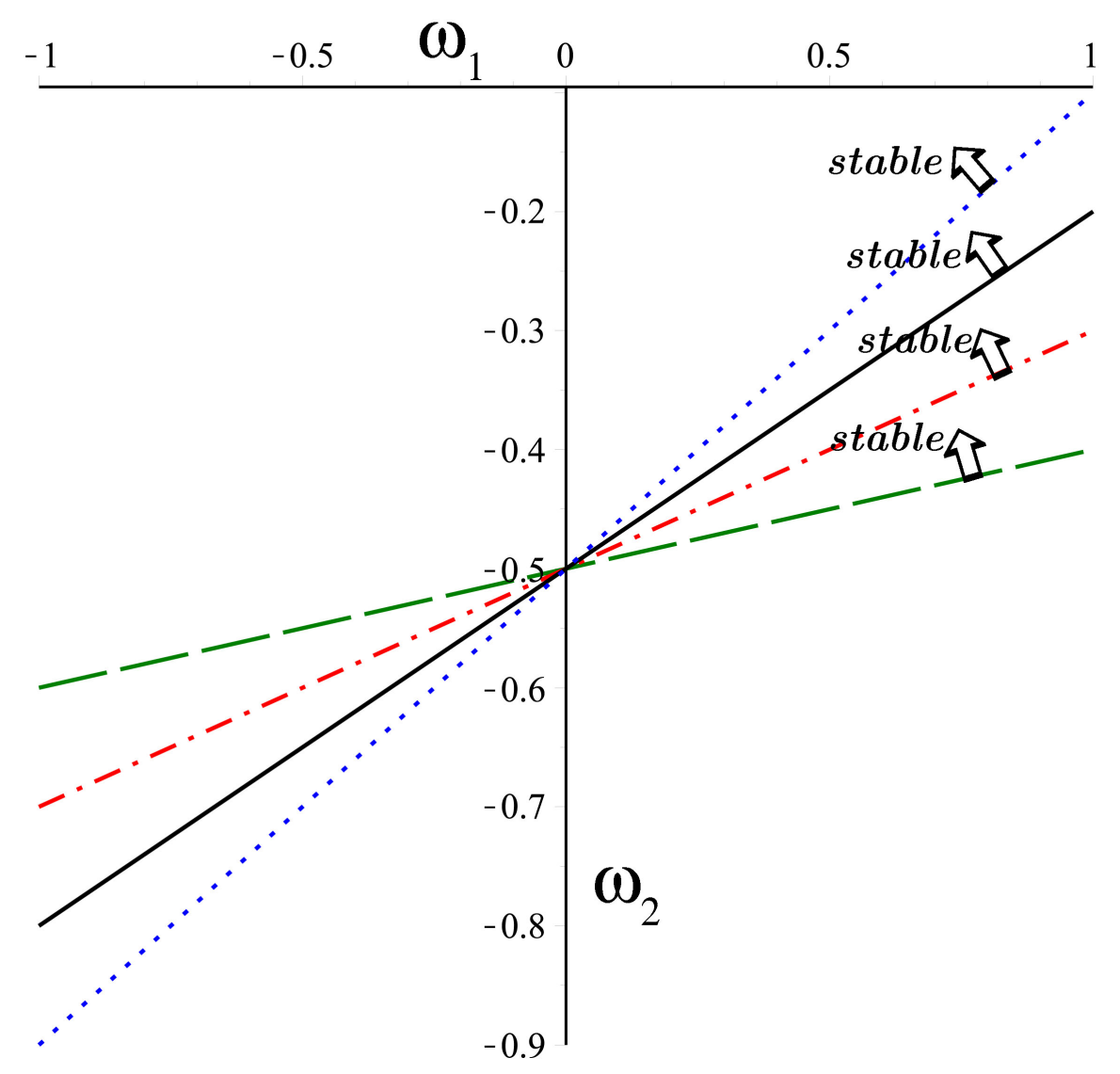

FIG. 2: A plot of $\omega_{2}$ with respect to $\omega_{1}$ for various values of $\chi=0.1,0.2,0.3$ and 0.4 . The arrows show the region of stability while the opposite side is the unstable zone for each case.

Before completing this section we would like to find the explicit form of the energy density $\sigma$ after the perturbation. In both examples we have worked out in this chapter we assumed $\frac{\partial \psi}{\partial R}=\omega_{1}$ and $\frac{\partial \psi}{\partial \sigma}=\omega_{2}$ in which $\omega_{1}$ and $\omega_{2}$ are two constants. Integration with respect to $R$ and $\sigma$ results in

$$
\psi=\omega_{1} R+\omega_{2} \sigma+C_{0}
$$


in which $C_{0}$ is an integration constant. As $p=\psi$ should give the equilibrium pressure at $R=R_{0}$ we can find the value of $C_{0}$ as

$$
C_{0}=p_{0}-\omega_{1} R_{0}-\omega_{2} \sigma_{0}
$$

and therefore the dynamic pressure becomes

$$
p=\omega_{1}\left(R-R_{0}\right)+\omega_{2}\left(\sigma-\sigma_{0}\right)+p_{0} .
$$

This EoS together with Eq. (19) gives the differential equation

$$
\frac{d \sigma}{d R}+\frac{2}{R}\left(\omega_{1}\left(R-R_{0}\right)+\omega_{2}\left(\sigma-\sigma_{0}\right)+p_{0}+\sigma\right)=0
$$

which must be satisfied by $\sigma$. The solution of this equation is given by

$$
\sigma(R)=\frac{\omega_{2} \sigma_{0}-p_{0}+\omega_{1} R_{0}}{1+\omega_{2}}-\frac{2 \omega_{1} R}{3+2 \omega_{2}}+\frac{C_{1}}{R^{2\left(\omega_{2}+1\right)}}
$$

in which $C_{1}$ is an integration constant. Imposing $\sigma\left(R_{0}\right)=\sigma_{0}$ yields

$$
C_{1}=R_{0}^{2\left(\omega_{2}+1\right)}\left(\frac{\sigma_{0}+p_{0}}{1+\omega_{2}}-\frac{\omega_{1} R_{0}}{3+5 \omega_{2}+2 \omega_{2}^{2}}\right)
$$

Finally the closed form of the energy density is found to be

$$
\sigma(R)=\frac{\omega_{2} \sigma_{0}-p_{0}+\omega_{1} R_{0}}{1+\omega_{2}}-\frac{2 \omega_{1} R}{3+2 \omega_{2}}+\left(\frac{R_{0}}{R}\right)^{2\left(\omega_{2}+1\right)}\left(\frac{\sigma_{0}+p_{0}}{1+\omega_{2}}-\frac{\omega_{1} R_{0}}{3+5 \omega_{2}+2 \omega_{2}^{2}}\right) .
$$

We note that at $R=R_{0}, \sigma(R)$ reduces to $\sigma_{0}$ and it is a function of $R$ as well as $\omega_{1}$ and $\omega_{2}$. The case $\omega_{1}=0$ admits

$$
\sigma(R)=\frac{\omega_{2} \sigma_{0}-p_{0}}{1+\omega_{2}}+\left(\frac{R_{0}}{R}\right)^{2\left(\omega_{2}+1\right)}\left(\frac{\sigma_{0}+p_{0}}{1+\omega_{2}}\right)
$$

while when $\omega_{2}=0$ we find

$$
\sigma(R)=-p_{0}+\omega_{1} R_{0}-\frac{2 \omega_{1} R}{3}+\left(\frac{R_{0}}{R}\right)^{2}\left(\sigma_{0}+p_{0}-\frac{\omega_{1} R_{0}}{3}\right) .
$$

In the case both $\omega_{1}$ and $\omega_{2}$ are set to zero the energy density becomes

$$
\sigma(R)=-p_{0}+\left(\frac{R_{0}}{R}\right)^{2}\left(\sigma_{0}+p_{0}\right)
$$

while

$$
p=p_{0}
$$

even after the perturbation. According to the Fig. 2, this is one of the case where the thin-shell is stable with

$$
V_{0}^{\prime \prime}=\frac{2 \Omega}{R_{0}^{2}}
$$

which is clearly positive.

\section{CONCLUSION}

We studied the stability of the timelike thin-shells in spherically symmetric spacetimes with a variable EoS on the shell of the form $p=\psi(\sigma, R)$, [23, 24]. We recall that in previous studies this was taken simply as $p=p(\sigma)$. We analyzed the stability of the thin-shells against a radial perturbation and by a linearized approximation we found a general condition to be satisfied in order to have a stable spherically symmetric thin-shell. We applied our results to 
two explicit cases with the variable EoS on the shell and numerically, as well as analytically we obtained the stability regions. Our first thin-shell joins two spacetimes of local cloud of strings, characterized by two distinct parameters $\kappa_{1}>0$ and $\kappa_{2}>0$ such that $\kappa_{1}>\kappa_{2}$. The second thin-shell connects a vacuum flat spacetime to the Schwarzschild metric. In both examples the weak energy conditions are satisfied for which the stability regions are identified and plotted.

[1] W. Israel. Nuovo Cimento B, 44:1, (1966).

[2] W. Israel. Nuovo Cimento B, 48:463, (1966).

[3] D. Kijowski, J. Magli. Gen. Relativ. Gravitation, 38:1697, (2006).

[4] C. Frauendiener, J. Hoenselaers and W. Konrad. Classical Quantum Gravity, 7:583, (1990).

[5] J. Brady, P. R. Louko and E. Poisson. Phys. Rev. D, 44:1891, (1991).

[6] B. G. Schmidt. Phys. Rev. D, 59:024005, (1998).

[7] P. Musgrave and K. Lake. Class.Quant.Grav., 13:1885, (1996).

[8] K. G. Zloshchastiev. Int.J.Mod.Phys. D, 8:549, (1999).

[9] M. Ishak and K. Lake. Phys. Rev. D, 65:044011, (2002).

[10] F. S. N. Lobo and P. Crawford. Class. Quant. Grav., 22:4869, (2005).

[11] J. Crisostomo and R. Olea. Phys. Rev. D, 69:104023, (2004).

[12] S. del. Crisostomo, J. Campo and J. Saavedra. Phys. Rev. D, 70:064034, (2004).

[13] E. Gravanis and S. Willison. Phys. Rev. D, 75:084025, (2007).

[14] R. J. Gleiser and M. A. Ramirez. Class. Quant. Grav., 26:045006, (2009).

[15] E. F. Eiroa and C. Simeone. Phys. Rev. D, 83:104009, (2011).

[16] H. Beauchesne and A. Edery. Phys. Rev. D, 85:044056, (2012).

[17] S. H. Mazharimousavi and M. Halilsoy. Eur. Phys. J. C., 73:2527, (2013).

[18] J. P. S. Lemos and G. M. Quinta. Phys. Rev. D, 88:067501, (2013).

[19] J. P. S. Lemos and G. M. Quinta. Phys. Rev. D, 89:084051, (2014).

[20] J. G. Pereira, J. P. Coelho and J. A. Rueda. Phys. Rev. D, 90:123011, (2014).

[21] S. H. Mazharimousavi and M. Halilsoy. Eur. Phys. J. C., 75:334, (2015).

[22] E. Eiroa, E. F. Rubin de Celis and C. Simeone. Eur. Phys. J. C., 76:546, (2016).

[23] N. M. Garcia, F. S. N. Lobo and M. Visser, Phys. Rev. D 86:044026, (2012).

[24] V. Varela Phys. Rev. D 92:044002, (2015).

[25] P. S. Letelier, Phys. Rev. D 20:1294 (1979).

[26] S. G. Ghosh and S. D. Maharaj, Phys. Rev. D 89:084027 (2014).

[27] S. G. Ghosh, U. Papnoi and S. D. Maharaj, Phys. Rev. D 90:044068 (2014).

[28] S. H. Mazharimousavi and M. Halilsoy, Eur. Phys. J. C 76:95 (2016). 